\title{
Metabolic classification of herb plants by NMR-based metabolomics
}

\author{
Hee-Eun Kim, , ${ }^{1, a}$ Ye Hun Choi, ${ }^{2, a}$ Kwang-Ho Choi, ${ }^{1, a}$ Ji Su Park, ${ }^{2}$ Hyeon Su Kim, ${ }^{2}$ \\ Jun Hyeok Jeon, ${ }^{2}$ Min Soo Heu, ${ }^{3}$ Dong-Seon Shin, ${ }^{2}$ and Joon-Hwa Lee ${ }^{1, *}$ \\ ${ }^{I}$ Department of Chemistry and Research Institute of Natural Science, Gyeongsang National University, \\ Jinju, Gyeongnam 660-701, Korea. \\ ${ }^{2}$ Gyeongnam Science High School, Jinju, Gyeongnam 660-851, Korea. \\ ${ }^{3}$ Department of Food and Nutrition and Institute of Marine Industry, Gyeongsang National University, \\ Jinju, Gyeongnam 660-701, Korea. \\ (Received Aug 10, 2012; Revised Sep 15, 2012; Accepted Sep 25, 2012)
}

\begin{abstract}
Metabolomics is the systematic identification and quantification of all metabolites in an organism or biological sample. NMR has been used as a major application tool in plant metabolomics such as quality control, chemotaxonomy, and analysis of genetically modified plants. Herbal medicines are the important therapeutics and are used to manage common diseases such as cold, inflammation, pain, heart diseases, liver cirrhosis, diabetes and central nerve system diseases. Herb plants include various kinds of species such as geranium, mint, and thyme and so on and contain different kinds of metabolites. We performed NMR-based metabolomics study on the seven different species of herb plants using ${ }^{1} \mathrm{H}$ NMR experiments and OPLS-DA to understand the correlation between the classification of herb plants and their metabolite contents. This study showed clear metabolic discrimination among various herb plants. This metabolmics study found several diagnostic NMR signals which are able to be used as bio-markers for identification of the specific herb plants among various species. Clear metabolic discrimination of herb plants suggests three chemotaxonomic groups of herb species.
\end{abstract}

Keywords : NMR, metabolomics, herb, plant, classification, chemotaxonomy

\footnotetext{
${ }^{a}$ These authors contributed equally to this work.

Department of Chemistry and Research Institute of Natural Science, Gyeongsang National University, Jinju, 660-701, Republic of Korea. email : joonhwa@gnu.ac.kr Journal of the Korean Magnetic Resonance Society 2012 December; 16(2): 91-102 http://dx.doi.org/10.6564/JKMRS.2012.16.2.091
} 


\section{INTRODUCTION}

Metabolomics is the systematic identification and quantification of all metabolite in an organism or biological sample. ${ }^{1,2} \mathrm{NMR}$ is a very suitable method to allow the detection of a wide range of metabolites with high robustness. ${ }^{2,3}{ }^{1} \mathrm{H}$ NMR has been used as a major application tool in plant metabolomics such as quality control, ${ }^{4}$ chemotaxonomy, ${ }^{5}$ and analysis of genetically modified plants. ${ }^{6}$ NMR-based metabolic profiling also could be applied to classification, characterization, and identification of plant-derived products., ${ }^{2,3}$

Herbal medicines are the important therapeutics and are used to manage chronic diseases. ${ }^{7}$ Most people in Asian countries use herbal medicines for promoting health and managing common diseases such as cold, inflammation, pain, heart diseases, liver cirrhosis, diabetes and central nerve system diseases. ${ }^{7}$ Herb plants include various kinds of species such as geranium, mint, and thyme and so on. Because each herb plant contains different kinds of metabolites, they should be used for a suitable purpose as herbal medicine. ${ }^{7}$ However, despite the importance of metabolite contents of herbal medicine, the chemotaxonomy of the herb species to identify the metabolic similarities between species are not clear yet.

We performed NMR-based metabolomics study on the seven different species of herb plants (Table 1) to understand the correlation between the classification of herb plants and their metabolite contents. Clear metabolic discrimination of herb plants in this study makes the chemotaxonomic 
classification of herb species possible.

\section{EXPERIMENTAL METHODS}

All herbal plants were purchased from Herb Web-mart (http://www.herb5.co.kr, Korea). The leaves of herb plants were well ground under liquid $\mathrm{N}_{2}$ condition and lyophilized. $0.75 \mathrm{~mL}$ of $\mathrm{CH}_{3} \mathrm{OH}-\mathrm{d}_{4}$ and $0.75 \mathrm{~mL}$ of $10 \mathrm{mM} \mathrm{KH}_{2} \mathrm{PO}_{4}$ buffer in $\mathrm{D}_{2} \mathrm{O}(\mathrm{pH}$ 6.0) containing 2-2-dimethyl-2silapentane-5-sulfonate (DSS) were added to $50 \mathrm{mg}$ of well-ground herb leaves. The mixture was ultrasonicated for $20 \mathrm{~min}$ at room temperature and centrifugated at 12,000 rpm for $10 \mathrm{~min}$. Each NMR sample consisted of $0.5 \mathrm{~mL}$ of supernatant, which was stored at $4{ }^{\circ} \mathrm{C}$.

Table 1. Sample lists of herb plants studied here.

\begin{tabular}{llc}
\hline Name & No. of samples \\
\hline Rose geranium & Pelargonium captitatum & 4 \\
Peppermint geranium & Pelargonium captitatum & 5 \\
Apple geranium & Pelargonium ordoratissimum & 3 \\
Lemon geranium & Pelargonium limoneum & 4 \\
Gold lemon thyme & Tymus X citriodorus aureus & 5 \\
Pineapple mint & Mentha species & 3 \\
Chocolate mint & Mentha piperita & 3 \\
\hline
\end{tabular}


All NMR experiments were performed on an Agilent DD2 700MHz NMR spectrometer (GNU, Jinju, Korea) equipped with a triple resonance probe at $25{ }^{\circ} \mathrm{C}$. Residual water signal of onedimensional (1D) NMR spectra was suppressed by using a pre-saturation water suppression pulse sequence and these data were processed with the program Chenomx NMR Suite 7.1 (Chenomx, Alberta, Canada) or FELIX2004 (Accelrys, San Diego, CA). DSS was used for the ${ }^{1} \mathrm{H}$ reference. All NMR spectra were phased and baseline-corrected with the program Chenomx NMR Suite 7.1 (Chenomx, Alberta, Canada). Principal component analysis (PCA) was carried out in SIMCA P+ version 12 (Umetrics, Kinnelon, NJ). Furthermore, orthogonal projection on latent structure-discriminant analysis (OPLS-DA) was used to analyze the NMR binning data with class informations. 8,9

\section{RESULTS AND DISCUSSION}

The NMR data binning was performed from 0 to $10 \mathrm{ppm}$ with a bin size of $0.04 \mathrm{ppm}$. The spectral region of the suppressed water $(4.60 \sim 5.00 \mathrm{ppm})$ and DSS $(2.80 \sim 2.88,1.72 \sim 1.82,0.54 \sim$ 0.64 , and $0 \sim 0.04 \mathrm{ppm}$ ) signals were excluded from the binning process. PCA was applied to the 1D NMR binning data in order to obtain the maximum variation between the samples. PCA with first seven components explaining $85 \%$ of the variance did not show clear differentiation among the herb samples from different classes, although some herb samples showed separation from other samples (Fig. 1). For example, the score plot (PC1 vs. PC2) in Fig. 1A shows that Apple geranium is clearly 
separated from other samples. Similarly, the PC4 and PC6 components well separate Pineapple mint from other samples (Fig. 1D). In addition, using PC1 and PC4, Gold lemon thyme and Pineapple mint are also well differentiated from other samples (Fig. 1B). However, Rose geranium, Lemon geranium, and Peppermint geranium cannot be separated from other herb plants (Fig. 1).

A

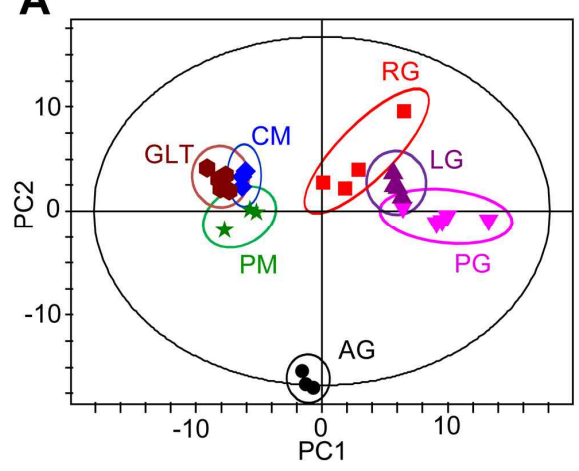

C

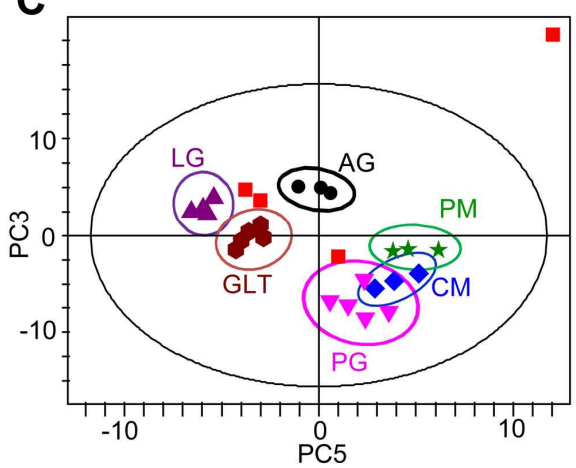

B

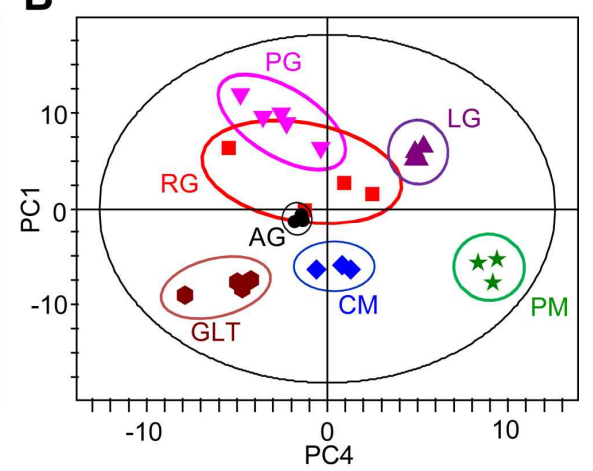

D

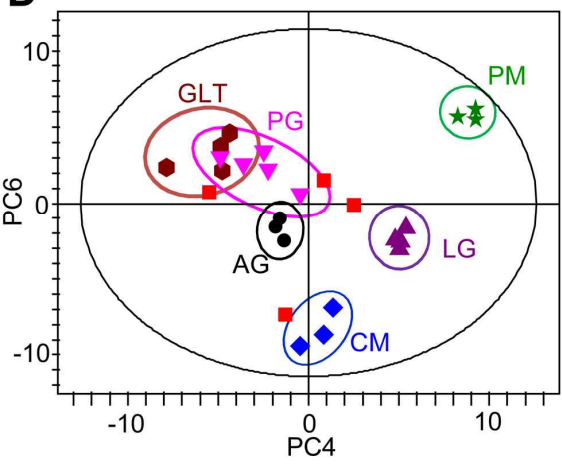

Figure 1. PCA score plots of (A) PC1 vs. PC2, (B) PC4 vs. PC1, (C) PC3 vs. PC5, and (D) PC4 vs. PC6 derived from ${ }^{1} \mathrm{H}$ NMR spectra of 27 herb extract samples from different species. RG: Rose geranium, PG: Peppermint geranium, AG: Apple geranium, LG: Lemon geranium, GLT: Gold lemon thyme, PM: Pineapple mint, CM: Chocolate mint. 
To maximize the differences between species, OPLS-DA model was constructed with six PLS components and two orthogonal components. $R 2 x$ values of six PLS components (OPLS1 $\sim$ OPLS6) are $19.8,16.9,12.0,9.5,7.3$, and $5.8 \%$, respectively. Similar to PCA, Apple geranium is clearly separated from other samples using OPLS1 and OPLS2 components (Fig. 2A). The loading plot obtained from OPLS-DA using OPLS1 and OPLS2 indicated that some NMR signals were diagnostics for identification of Apple geranium among various herb plants (circle in Fig. 3A). For example, the ${ }^{1} \mathrm{H}$ NMR peaks at 3.81 and $4.32 \mathrm{ppm}$ in Apple geranium have much stronger intensities compared to those in other herb samples (Fig. 4). The relative peak intensities of these two resonances centered at 3.81 and $4.32 \mathrm{ppm}$ in Apple geranium are 6-times higher than those in other herb samples (Table 2).

Table 2. Relative proton amounts $(\%)^{\mathrm{a}}$ of bio-markers of herb extracts.

\begin{tabular}{lllllllll}
\hline Marker & $\begin{array}{l}\text { Chemical } \\
\text { shift } \\
\text { (ppm) }\end{array}$ & $\begin{array}{l}\text { Rose } \\
\text { geranium }\end{array}$ & $\begin{array}{l}\text { Pepper- } \\
\text { mint } \\
\text { geranium }\end{array}$ & $\begin{array}{l}\text { Apple } \\
\text { geranium }\end{array}$ & $\begin{array}{l}\text { Lemon } \\
\text { geranium }\end{array}$ & $\begin{array}{l}\text { Gold } \\
\text { lemon } \\
\text { time }\end{array}$ & $\begin{array}{l}\text { Pineapple } \\
\text { mint }\end{array}$ & $\begin{array}{l}\text { Chocolate } \\
\text { mint }\end{array}$ \\
\hline GLT-1 & 9.87 & 0 & 0 & 0 & 0 & 0.02 & 0 & 0 \\
GLT-2 & 9.68 & 0 & 0 & 0 & 0 & 0.01 & 0 & 0 \\
LG-1 & 8.68 & 0.01 & 0 & 0 & 0.05 & 0 & 0 & 0 \\
CM-1 & 6.21 & 0.03 & 0.22 & 0.04 & 0.04 & 0.10 & 0.02 & 1.16 \\
PG-1 & 5.27 & 0.16 & 0.43 & 0.21 & 0.08 & 0.06 & 0.06 & 0.08 \\
AG-1 & 4.32 & 0.97 & 0.51 & 5.18 & 0.61 & 1.22 & 0.98 & 0.88 \\
AG-2 & 3.81 & 1.25 & 1.81 & 11.05 & 1.57 & 2.36 & 1.52 & 1.49 \\
PM-1 & 2.07 & 0.76 & 0.38 & 0.37 & 0.46 & 1.00 & 4.86 & 0.96 \\
\hline
\end{tabular}




\begin{tabular}{lllllllll}
\hline PM-2 & 1.85 & 0.45 & 0.43 & 0.26 & 0.33 & 0.52 & 4.33 & 0.53 \\
PM-3 & 1.48 & 1.22 & 0.89 & 0.57 & 1.01 & 1.21 & 4.85 & 1.11 \\
CM-2 & 1.18 & 2.01 & 0.79 & 0.55 & 1.67 & 1.55 & 0.74 & 5.06 \\
PG-2 & 0.98 & 0.83 & 1.78 & 0.35 & 0.74 & 0.55 & 0.77 & 1.21 \\
PG-3 & 0.92 & 2.56 & 4.11 & 1.07 & 2.20 & 1.18 & 1.02 & 3.26 \\
\hline
\end{tabular}

${ }^{\mathrm{a}}$ Relative proton amounts $(\%)=$ peak intensity $/ \Sigma($ peak intensities $) \times 100$.

The score plot (OPLS4 vs. OPLS1) in Fig. 2B shows that Pineapple mint and Gold lemon thyme are clearly separated from other samples. The OPLS-DA loading plot using OPLS4 and OPLS1 indicated that some NMR signals were diagnostics for identification of Pineapple mint and Gold lemon thyme among various herb plants (circles in Fig. 3B). The three ${ }^{1} \mathrm{H}$ NMR peaks centered at $1.50,1.85$, and $2.07 \mathrm{ppm}$ in Pineapple mint show relatively stronger than those of other herb samples (Fig. 4 and Table 2). Similarly, the two ${ }^{1} \mathrm{H}$ NMR peaks centered at 9.68 and 9.87 ppm in Gold lemon thyme have significantly stronger intensities compared to those of other herb samples (Fig. 4 and Table 2).

In contrast to PCA, Chocolate mint and Peppermint geranium are also able to be separated from other samples using OPLS5 and OPLS3 components (Fig. 2C). The OPLS-DA loading plot using OPLS5 and OPLS3 provides diagnostic NMR signals for identification of Chocolate mint and Peppermint geranium among various herb plants (circles in Fig. 3C). The ${ }^{1} \mathrm{H}$ NMR peaks at 1.18 and $6.21 \mathrm{ppm}$ in Chocolate mint show much stronger intensities than those of other herb samples (Fig. 4 
and Table 2). The extracts from Peppermint geranium exhibit relatively stronger ${ }^{1} \mathrm{H}$ NMR peaks at 0.92, 0.98, and 5.27 ppm compared to other herb plants (Fig. 4 and Table 2).

A

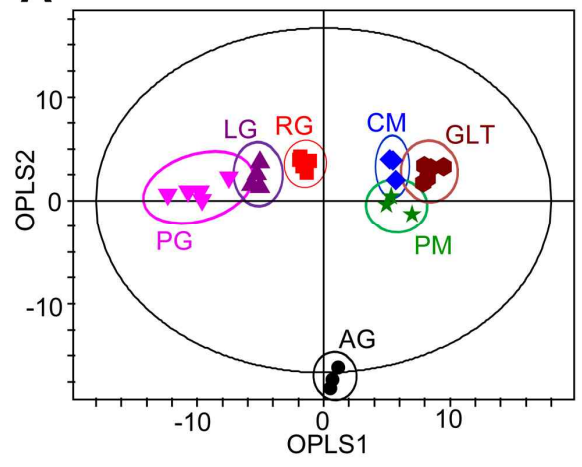

C

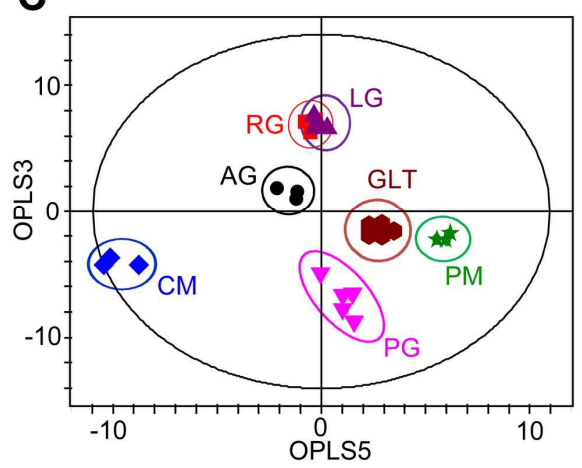

B

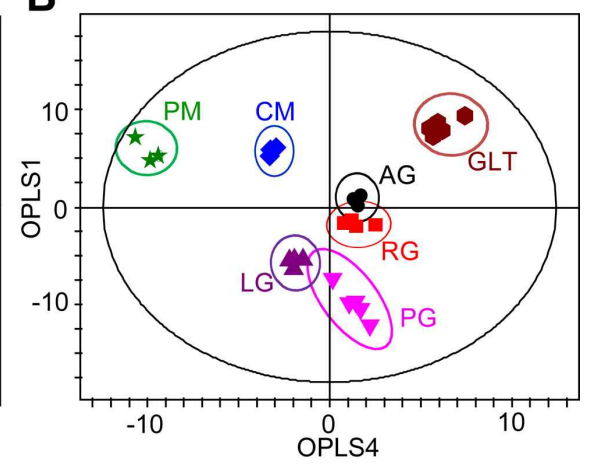

D

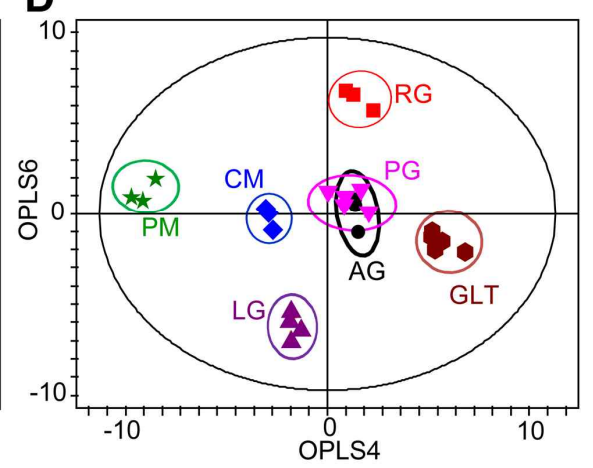

Figure 2. OPLS-DA score plots of (A) OPLS1 vs. OPLS 2, (B) OPLS 4 vs. OPLS1, (C) OPLS3 vs. OPLS5, and (D) OPLS4 vs. OPLS6 derived from ${ }^{1} \mathrm{H}$ NMR spectra of 27 herb extract samples from different species. RG: Rose geranium, PG: Peppermint geranium, AG: Apple geranium, LG: Lemon geranium, GLT: Gold lemon thyme, PM: Pineapple mint, CM: Chocolate mint. 
A

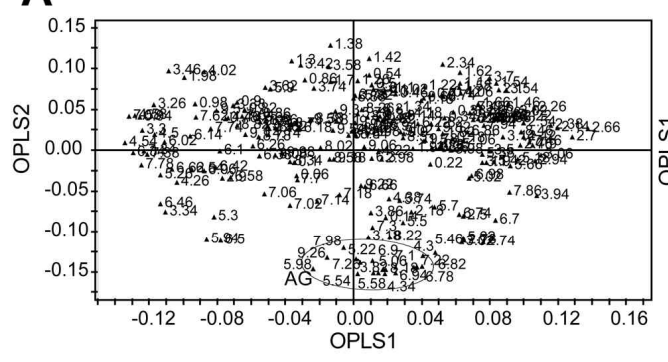

B

\section{C}
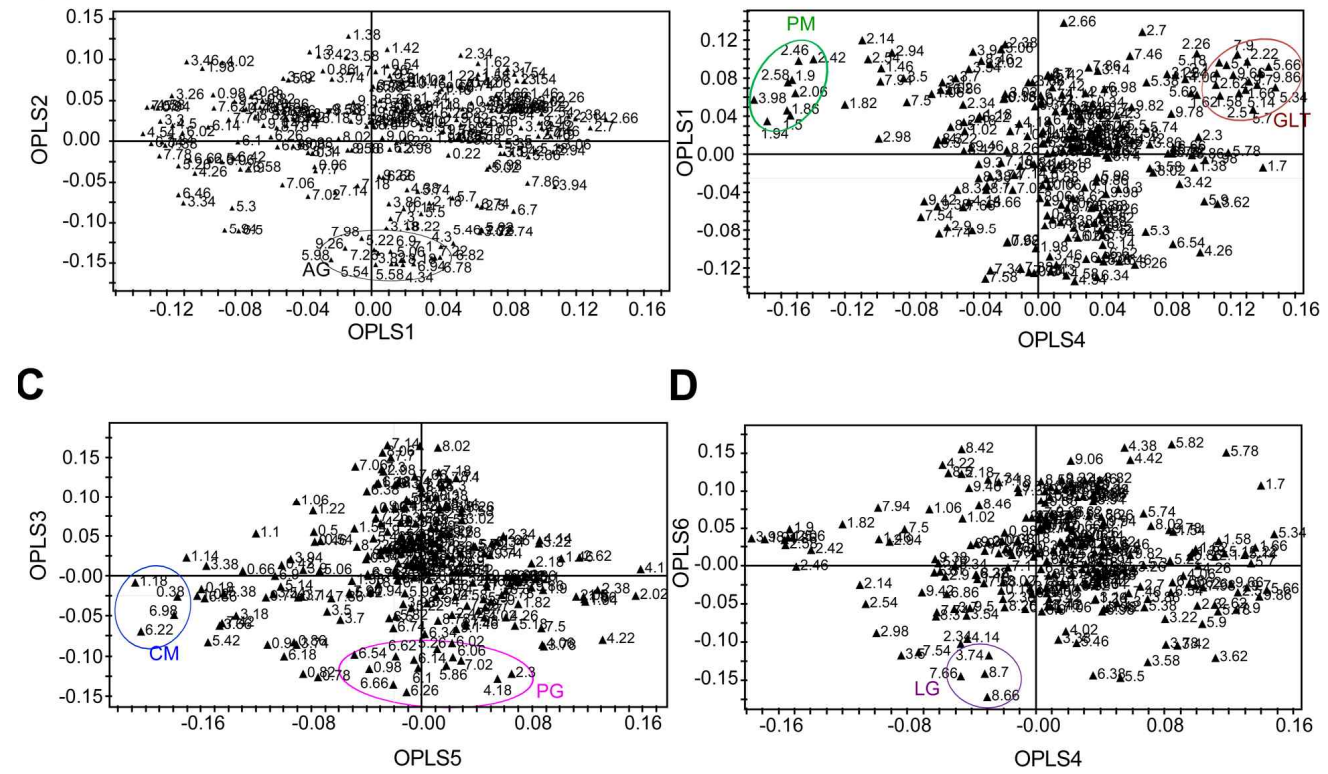

D

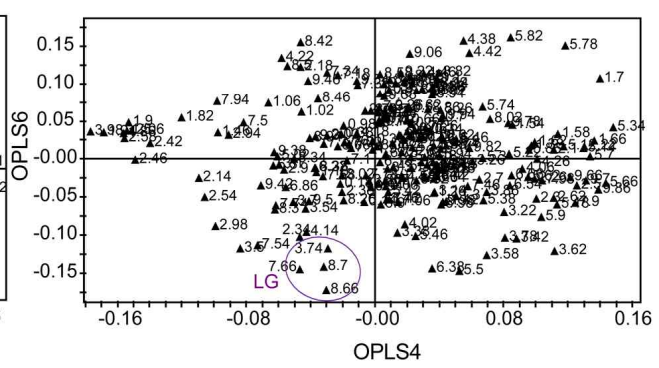

Figure 3. OPLS-DA loading plots of (A) OPLS1 vs. OPLS 2, (B) OPLS 4 vs. OPLS1, (C) OPLS3 vs. OPLS5, and (D) OPLS4 vs. OPLS6 derived from ${ }^{1} \mathrm{H}$ NMR spectra of 27 herb extract samples from different species. RG: Rose geranium, PG: Peppermint geranium, AG: Apple geranium, LG: Lemon geranium, GLT: Gold lemon thyme, PM: Pineapple mint, CM: Chocolate mint.

The score plot (OPLS4 vs. OPLS6) in Fig. 2D shows that Lemon geranium and Rose geranium are well separated from other samples. This study found a diagnostic NMR signal (at $8.68 \mathrm{ppm}$ ) for identification of Lemon geranium among various herb plants (Fig 4 and Table 2). However, we cannot find any diagnostic NMR signals for identification of Rose geranium because the ${ }^{1} \mathrm{H}$ NMR spectra for the extract samples from Rose geranium are very similar to those of Lemon geranium (data not shown). 


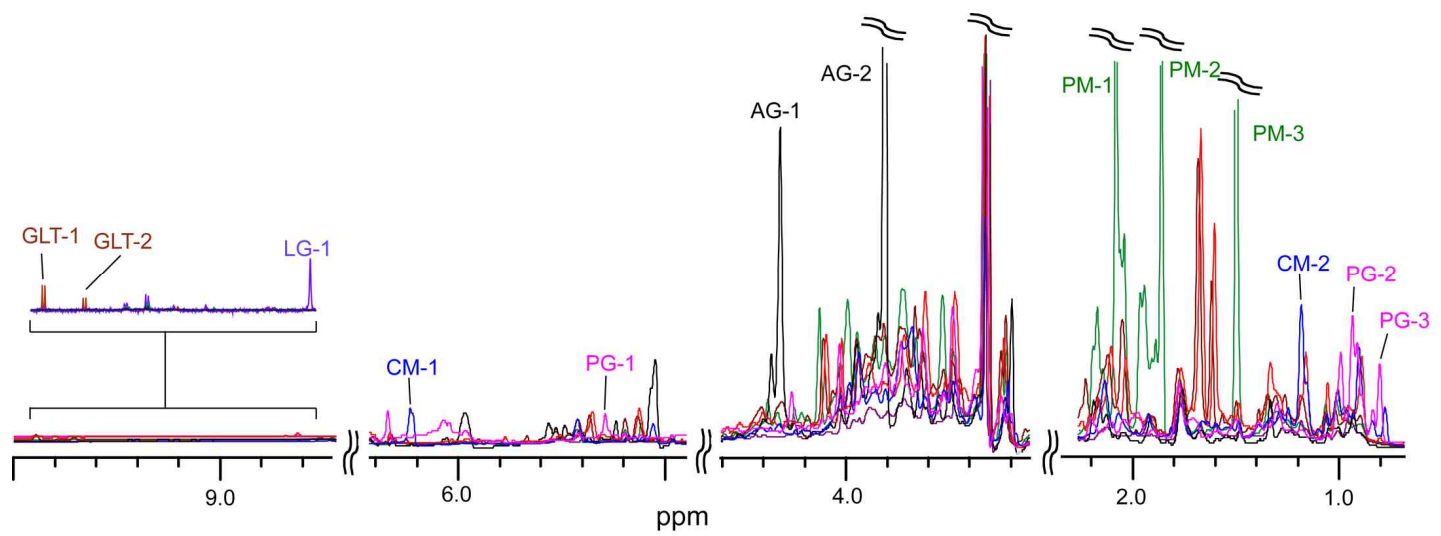

Figure 4. Expanded regions of $1 \mathrm{H}$ NMR spectra of the herb extracts from Rose geranium, Peppermint geranium, Apple geranium, Lemon geranium, Gold lemon thyme, Pineapple mint, and Chocolate mint determined at $25^{\circ} \mathrm{C}$.

Based on OPLS-DA, hierarchical cluster analysis (HCA) was performed to obtain further information on the metabolic similarities and differences among herb species. As shown in Fig. 5, the following three groups were obtained from HCA. The metabolomes of Chocolate mint and Pineapple mint were found to be very similar to each other (Fig. 5). In addition, Gold lemon thyme can be grouped with two mint plants based on their metabolomes (Fig. 5). Thus, the first group consists of two mints (Chocolate mint and Pineapple mint) and Gold lemon htyme. As expected, the metabolome of Rose geranium and Lemon geranium were found to be similar to that of Peppermint geranium (Fig. 5). These three geranium plants constitute the second group of herb plants. Surprisingly, the metabolome of Apple geranium was found to be significantly different from not only other geranium plants but also mint plants (Fig. 5). Thus, Apple geranium is thought to be the third group in HCA in this study. 


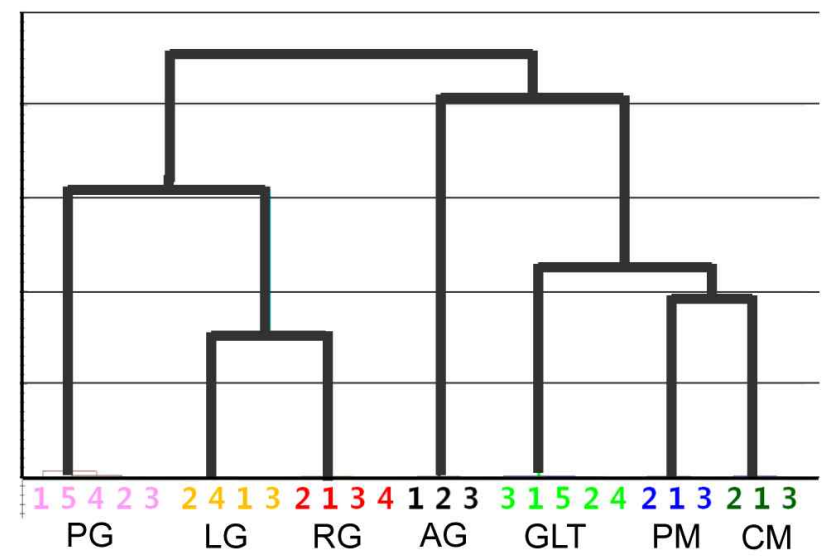

Figure 5. Dendrogram of HCA results based on OPLS-DA. RG: Rose geranium, PG: Peppermint geranium, AG: Apple geranium, LG: Lemon geranium, GLT: Gold lemon thyme, PM: Pineapple mint, CM: Chocolate mint.

In summary, we performed metabolomics study on the seven different species of herb plants study using ${ }^{1} \mathrm{H}$ NMR experiments and OPLS-DA. This study showed clear metabolic discrimination among various herb plants. This metabolmics study found several diagnostic NMR signals which are able to be used as bio-markers for identification of the specific herb plants among various species. HCA suggests three groups of herb plants based on their metabolomes.

\section{Acknowledgment}

This work was supported by the KOFAC Science High School R\&E Program, the NRF Grants (2010-0014199, NRF-C1ABA001-2010-0020480) funded by Korean Government (MEST). This 
work was also supported by a grant from Next-Generation BioGreen 21 Program (SSAC, No. PJ009041), Rural Development Administration, Korea. This work was also supported by Interdisciplinary Research Promotion Program of College of Natural Science, GNU. We thank the GNU Central Instrument Facility for performing NMR experiments.

\section{REFERENCES}

1. J. Schipsema, Phyrochem. Anal. 21, 14. (2009).

2. H. K. Kim, Y. H. Choi, R. Verpoorte, Nat. Protoc. 5, 536. (2010).

3. H. K. Kim, Y. H. Choi, R. Verpoorte, Trends. Biotechnol. 29, 267. (2011).

4. B. Rasmussen, O. Cloarec, H. Tang, D. Staerk, J. W. Jaroszewski, Planta Med. 72, 556. (2006).

5. H. K. Kim, Saifullah; S. Khan, E. G. Wilson, S. D. P. Kricun, A. Meissner, S. Goraler, A. M. Deelder, Y. H. Choi, R. Verpoorte, Phytochemistry 71, 773. (2010).

6. E. Barros, S. Lezar, M. J. Anttonen, J. P. van Dijk, R. M.;Rohlig, E. J. Kok, K.-H. Engel, Plant Biotechnol. J. 8, 436. (2010).

7. Y. H. Choi, Y.-W. Chin, Y. G. Kim, Arch. Pharm. Res. 34, 1843. (2011).

8. Y. Jung, Y.-S. Jung, G.-S. Hwang, J. Korean Magn. Reson. Soc. 15, 90. (2011).

9. J. Kim, J. Park, S.-S. Park, G.-S. Hwang, J. Korean Magn. Reson. Soc. 16, 54. (2012). 